\title{
A Self-organized Dynamic Clustering method and its Multiple Access Mechanism for Multiple WBANs
}

\author{
Jiasong $\mathrm{Mu}$, Robert Stewart, Liang Han and David Crawford
}

\begin{abstract}
Due to its wide application range and attractive features, Wireless Body Area Networks (WBAN) is considered as a revolutionary technology which is envisaged to change how people manage and think about their health and their life styles. In this paper, we propose a Self-organized Dynamic Clustering (SDC) method and its multiple access mechanism to mitigate the interference and improve the QoS in multiple WBANs environment. To the best of our knowledge, this is the first paper which focuses on the spectrum allocation for multiple WBANs. We borrow the concepts of cell and cluster from cellular networks to allocate the channels for different WBANs. The clustering is self-organized to improve the data transmission for intra-WBAN communication by the information exchange via inter-WBAN communication. Additionally, based on the cluster architecture, an inter-WBAN relaying (IWR) protocol for packets with low privacy or high reliability is also investigated. The simulation results show that SDC has better signal to interference ratio compared with existing framework. Besides, SDC and IWR also provide better QoS performance in terms of higher data packet delivery ratio and lower packet delay.
\end{abstract}

Key words - Clustering, Interference mitigation, Spectrum allocation, WBAN

\section{INTRODUCTION}

$\mathrm{T}_{\mathrm{H}}^{\mathrm{H}} \mathrm{Nen}$ HANKS to the recent advances in Information and Communication Technologies (ICTs), the design of intelligent physiological sensor nodes is trending smaller size and more efficient in data sensing[1]. This progress facilitates the development of Wireless Body Area Networks (WBAN). WBAN typically consists of a collection of lowpower, miniaturized, invasive or non-invasive, lightweight devices with wireless communication capabilities placed in or on the human body for use in monitoring body vital and physiological data and immediate environment [2].

As its most attractive benefit is the capability of continuous functioning without obstructing user comfort in performing daily activities [3], WBAN is regarded as a revolutionary technology in various applications in health-care [4], fitness [5], smart cities [6], and many other compelling Internet of Things (IoT) applications [7], [8]. Especially, it may enhance the healthcare system significantly by reducing the cost, providing more comprehensive data and improve the mobility of the user [9].

Based on their roles in the networks, WBAN devices can be categorized into three types: a) Hub (aka sink, coordinator or gateway) organizes the network and acts as the

- Jiasong Mu is with the Tianjin Key Laboratory of Wireless Mobile Communications and Power Transmission, College of Electronic and Communication Engineering, Tianjin Normal University, Tianjin, China. E-mail: mujiasong@163.com

- Robert Stewart is with the Dept of Electronic and Electrical Engineering University of Strathclyde, Scotland, UK. E-mail: r.stewart@strath.ac.uk

- Liang Han is with the with the Tianjin Key Laboratory of Wireless Mobile Communications and Power Transmission, College of Electronic and Communication Engineering, Tianjin Normal University, Tianjin, China. Email:mujiasong@163.com.

- David Crawford is with the Dept of Electronic and Electrical Engineering, University of Strathclyde, Scotland, UK. E-mail: david.craw-

ford@strath.ac.uk gateway to the outside world; b) Router is intermediate nodes which have a parent node and a few child nodes through which they relay messages; c) End Node is only capable of performing its sensing task and communicating with its parent (a router) [10]. A typical WBAN is shown in Fig.1. Whereas, the communication in the figure only includes the intra-body communication which is the Tier 1 of a common 3-tier telemedicine system structure which cover multiple WBANs as shown in Fig.2. Tier 1 encompasses the individual intra-body communication, tier 2 denotes the extra-body communication between the different WBANs and the Internet which is performed by the hub and tier 3 represents the extra-body communication from the Internet to the medical server [11].

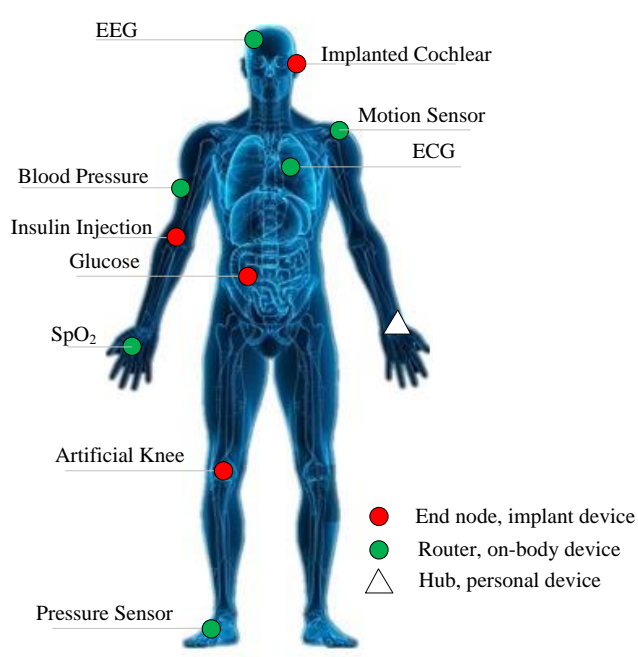

Fig.1. An example of WBAN 


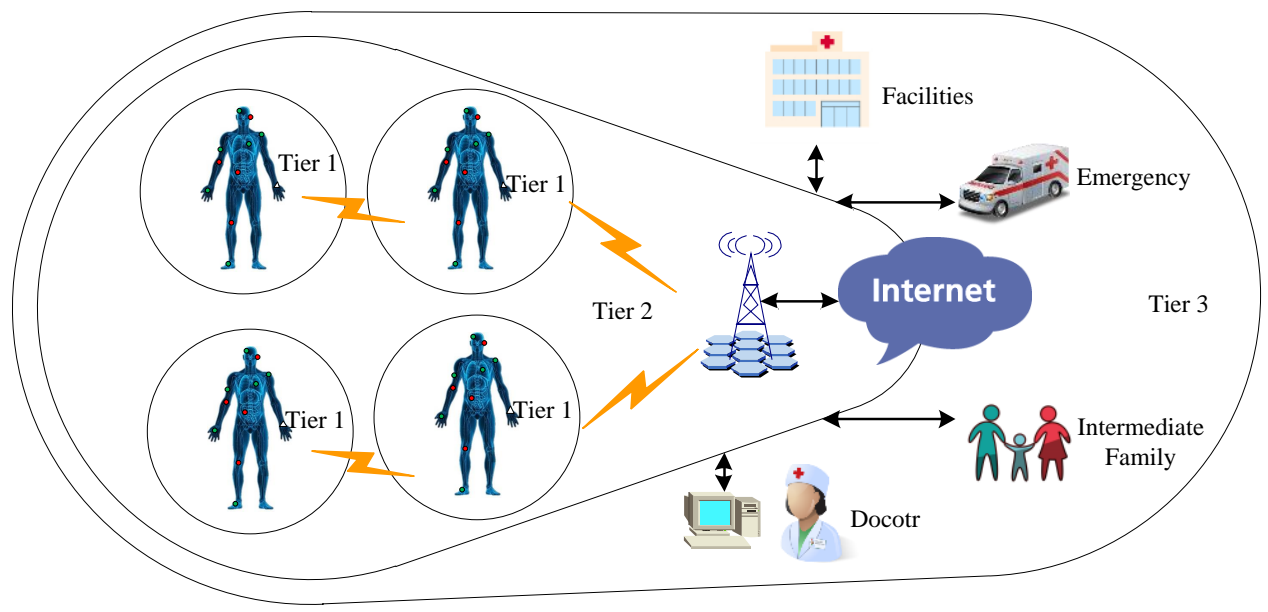

Fig. 2. Communication tiers for WBANs

Since there are several obvious differences between WBAN and conventional wireless technologies as shown in Fig.3 [12], [13], it brings new challenges in network design. WBAN technology is still in its primitive stage and needs further study [14]. As the candidates for the WBAN protocol, Bluetooth 4.0 [15], IEEE 802.15.4 [16] cannot fully satisfy the manifold WBAN application requirements [17]. Particular attention is paid to the IEEE 802.15.6, a communication standard optimized for low power devices and operation on, in or around the human body to serve a variety of applications including medical, consumer electronics, personal entertainment and other [18]. However, a considerable part of the existing deployments and studies are not fitting in the standard [19], [20]. Based on IEEE 802.15.6, the QoS requirement for different healthcare applications is shown in Table.1. Note that the spectrum for different applications is not specified in the standard, each sensor may use an exclusive channel or a shared one with other nodes. The available frequency bands in IEEE 802.15.6 is shown in Table 2. From the viewpoint of channels, one user may be involved in networks which are working in different spectrum. However, because all these networks are managed by a common hub device, to avoid confusion, one WBAN refers to the networks related to one hub/user in the following of this paper.

To guarantee the QoS for heterogeneous nodes, the
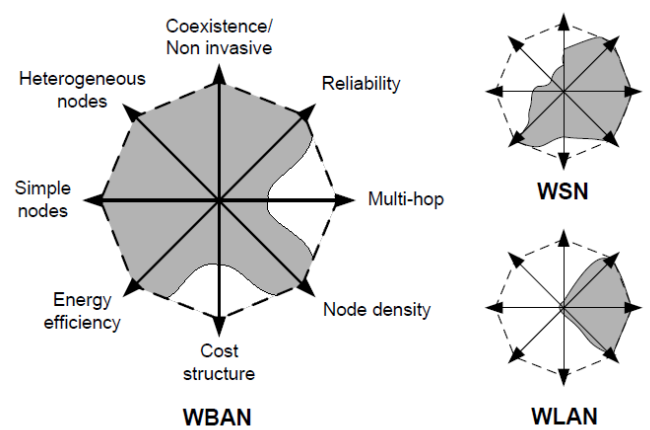

Fig. 3. Characteristics of WBAN compared Wireless Sensor Network (WSN) and Wireless Local Area Network (WLAN)
TABLE 1

QOS REQUIREMENTS FOR SOME WBAN APPLICATIONS [21]

\begin{tabular}{lccc}
\hline \hline \multicolumn{1}{c}{ Application Type } & Bit rate & Delay & BER \\
\hline Deep brain stimulation & $<320 \mathrm{kbps}$ & $<250 \mathrm{~ms}$ & $<10^{-10}$ \\
Drug delivery & $<16 \mathrm{kbps}$ & $<250 \mathrm{~ms}$ & $<10^{-10}$ \\
Capsule endoscope & $1 \mathrm{Mbps}$ & $<250 \mathrm{~ms}$ & $<10^{-10}$ \\
ECG & $192 \mathrm{kbps}$ & $<250 \mathrm{~ms}$ & $<10^{-10}$ \\
EEG & $86.4 \mathrm{kbps}$ & $<250 \mathrm{~ms}$ & $<10^{-10}$ \\
EMG & $1.536 \mathrm{Mbps}$ & $<250 \mathrm{~ms}$ & $<10^{-10}$ \\
Glucose level monitor & $<1 \mathrm{kbps}$ & $<250 \mathrm{~ms}$ & $<10^{-10}$ \\
Audio streaming & $1 \mathrm{Mbps}$ & $<20 \mathrm{~ms}$ & $<10^{-5}$ \\
Video streaming & $<10 \mathrm{Mbps}$ & $<100 \mathrm{~ms}$ & $<10^{-3}$ \\
Voice & $50-100 \mathrm{kbps}$ & $<100 \mathrm{~ms}$ & $<10^{-3}$ \\
\hline \hline
\end{tabular}

TABLE 2

FREQUENCY BANDS, BANDWIDTH FOR IEEE 802.15.6.

\begin{tabular}{cccc}
\hline \hline Description & Frequency Band & Bandwidth & Description \\
\hline Human Body & $16 \mathrm{MHz}$ & $4 \mathrm{MHz}$ & Implant \\
Communication & $27 \mathrm{MHz}$ & $4 \mathrm{MHz}$ & Implant \\
\hline \multirow{4}{*}{ Narrowband } & $402 \sim 405 \mathrm{MHz}$ & $300 \mathrm{KHz}$ & Implant \\
\cline { 2 - 4 } Communication & $420 \sim 450 \mathrm{MHz}$ & $300 \mathrm{KHz}$ & On-body \\
& $863 \sim 870 \mathrm{MHz}$ & $400 \mathrm{KHz}$ & On-body \\
& $902 \sim 928 \mathrm{MHz}$ & $500 \mathrm{KHz}$ & On-body \\
\cline { 2 - 4 } & $950 \sim 956 \mathrm{MHz}$ & $400 \mathrm{KHz}$ & On-body \\
\cline { 2 - 4 } & $2300 \sim 2400 \mathrm{MHz}$ & $1 \mathrm{MHz}$ & On-body \\
UWB & $2400 \sim 2438.5 \mathrm{MHz}$ & $1 \mathrm{MHz}$ & On-body \\
Communication & $3.2 \sim 4.7 \mathrm{GHz}$ & $499 \mathrm{MHz}$ & On-body \\
\hline \hline
\end{tabular}

Narrowband on-body communication bands are classified into 3 groups based on the similarity of their frequencies and modulation methods, as separated by grey lines.

QoS-based routing and the interference mitigation are of great importance in WBAN [22]. The existing studies on QoS-aware routing protocol mostly assume all the WBAN sensors use the same frequency to communicate [23]. it is not efficient from the view of resources efficiency, thus not 
practical. Moreover, the main difficulty in WBAN QoS improvement is the limited and changeful choices of the forwarding nodes due to the large attenuation for the electromagnetic wave propagation in or near the human body and the postural mobility. Some data in WBAN is of high privacy (such as medical application), while some data is not (such as the entertainment application) and should be allowed to be relayed by devices from other WBANs if doing so may increase the network performance. Thus, the clustering mechanism for multiple WBANs is needed to enable the inter-body transmission among sensors.

On the other hand, WBANs trend to be large density due to its individual-based deployment. Based on the requirement specified in IEEE 802.15.6 [16], up to 10 randomly distributed, co-located WBANs should be supported by the physical layer in a $6 \mathrm{~m}^{3}$ cube. This may provide new candidates for data forwarding. However, the cochannel interference may bring unneglectable effects on QoS in such conditions. Only few works have been concerned with a global solution for tens or hundreds of users, who are confined to a relatively small environment [24]. The most straightforward method to minimize the interference is to distance the nodes which use the same subchannel away from each other, like it is done in the cellular network. However, the cluster in mobile communication is formed based on fixed infrastructure, the base station, which is lacking in WBAN. Hence the clustering in WBAN needs to be redesigned.

In this paper, we propose a self-organized dynamic clustering method and its multiple access mechanism for multiple WBANs. To the best of our knowledge, this is the first paper addressing the issue of spectrum and channel access allocation for multiple WBANs. The aim of our work is to mitigate the co-channel interferences and improve the heterogeneous QoS for the WBAN communication with the coexistence of other users, especially for the large density environment. To better describe our work, we borrow the concept of cell and cluster from cellular networks. Our work has three major contributions. Firstly, the clustering is self-organized by the hubs which have more sufficient resources and relies on the tier 2 communication. We design both the FDMA and TDMA methods to improve the spectrum allocation. Secondly, the clustering enables the inter-body packet forwarding for the data with less security or high priority. This may reduce the node energy consumption and improve the QoS. However, the routing algorithm design is beyond the study in this paper. We just simply describe the principle for the next hop selection. Thirdly, the architecture is mainly based on the IEEE 802.15.6 for the tier 1 communication, which provides a good compatibility.

The remainder of the paper is organized as follows: Section 2 gives a brief review of the existing related work. The network architecture was discussed Section 3. In Section 4 the Self-organized Dynamic Clustering and its multiple access mechanism is proposed. The simulation results on different scenarios are shown in Section 5. Finally, Section 6 concludes the paper with the visions of further research.

\section{RELATED WORKS}

Existing studies on the QoS improvement in WBAN mostly focus on the QoS-based routing improvement. In [25], a QoS-aware routing protocol for biomedical sensor networks is proposed to improve the QoS by using different routing methods based on the data priority. This protocol was a proactive method, in which each node maintained a routing table which stores the current and possible routing to the hub. The simulation result showed that the QoS for different applications was improved, especially for the one with high priority. However, as the study in the early stage, the energy efficiency for the routing table maintenance caused by the mobility of human body was not taken into consideration. Djamel and Balasingham [26] proposed a LOCALMOR protocol for biomedical application. By using diverse techniques for packets with different QoS metric level, which covered the reliability, latency and residual energy in sensor nodes and transmission power between sensor nodes, the QoS was improved in localized and distributed network networks. Nevertheless, the scalability was a serious restriction for the algorithm. In [27], a prototype of WBANs, including virtual machine (VM) and virtualized cloudlet (VC) has been proposed for large scale WBAN simulation. By designing the storage and processing infrastructure, the simulation results showed that the power cost and the latency were effectively reduced by increasing the number of VMs and VCs. Since the performance of the proposed method was based on the function of external device, it did not improve the WBAN communication intrinsically. The data-centric routing was also studied as shown in [28], a DMQoS routing in data-centric architecture was designed to reduce the delay and improve the reliability. The traffic was categorized to provide customized QoS services by using adaptive modulation method. Although it outperformed other existing routing protocol, the QoS performance and geographic location information required in the next hop selection would be a huge cost for the simple WBAN device.

However, above methods only improve the data transmission in single WBAN, the interferences from the nodes in other WBANs are not considered, which is a bigger cause for the QoS degeneration. Only few works address the issue of interference mitigation and channel resources allocation in multiple WBANs. In [22], the authors studied several simple interference mitigation schemes which are affordable for WBAN devices, such as adaptive modulation, adaptive data rate and adaptive duty cycle, and the effectiveness of each method is evaluated by the Interference Mitigation Factor which was designed for different parameters. This paper gave a comprehensive evaluation for different mitigation method. However, the mentioned methods were at the node level and only single WBAN was analyzed. A priority-based allocation of time slots was proposed in [29]. To characterize the criticality of packets and some important properties of local data processing unit (LDPU) in data transmission, the author designed a fitness parameter, based on which the constant model hawk-dove game was introduced to allocate the access time proportionally for each node. The simulation results showed that the proposed method made a more reasonable distribution 
for the time slots by reducing the number of transmitting LDPUs, while the scheme only focused on the communication resources allocation in the time domain in a single WBAN. The work in [30] focused on the cooperation communication for multiple mobile WBANs without coordination between WBANs. A decode-and-forward protocol, selection combing and the time-division-multiple-access schemes were used to improve the signal-to-interferenceplus-noise-ratio (SINR). In the study, both the large-scale shadowing and small-scale fading were included to simulate the realistic channel in dynamic environments. By using a multi hop topology which were composed of two dual-hop links and two relays, better co-channel mitigation was achieved. Whereas, the SINR was improved by lower transmission power of nodes which was capacitated by the shorter transmission distance in multi hop topology which was not allowed in IEEE 802.15.6 standard. Additionally, only the TDMA techniques were considered but not the FDMA. Silva and etc [31] conduct a preliminary research on the impact of inter-user interference and quantified its variation with different number of networks and the data rates. Then, to alleviate the co-channel interference, the authors also proposed a system model in which a fixed network infrastructure was introduced to monitor the overall communication and manage the modulation method selection for each device in several WBANs. However, besides the dependency on fixed external devices and the assumption of the same band within a single WBAN, the neglect of spectrum allocation in different WBANs limited the development of the architecture.

\section{NETWORK ARCHITECTURE}

In the three-tire WBAN communication, as the tier- 3 belongs to the field of internet and big data processing, our architecture only covers the first two which refer to the intra and inter WBAN communication. To better describe our work, we borrow the concept of cell and cluster from cellular networks.

\subsection{Device types}

The WBAN nodes are classified into implant (in-body) node, body surface (on-body) node and external body node in terms of their implementations. They refer to the device implanted, placed no more than $2 \mathrm{~cm}$ from human body and farther away, separately. In our work, the external node is not considered since it is not a typical WBAN device.

Different form conventional wireless sensors, in-body nodes have a stringent requirement on thermal control, because the heat produced due to wireless communication and the power dissipation by the sensor circuitry [32], [33] may cause thermal damage to the human tissue inside the body if the communication lasts for a long time which even be life-threatening [34]. On the other hand, owing to the severe penetration loss for high frequency electromagnet wave, which is approximately $10 \mathrm{~dB}$ for $10 \mathrm{~mm}$ tissue penetration [35], Human Body Communication which uses electrostatic field communication is always preferred for the intra-body communication. Even if the wireless method is used, a unique low frequency band is allocated as shown in Table.2. For the reasons mentioned above, we regard the in-body nodes as end nodes in our architecture to minimize the power consumption and temperature rising. We assume the implanted end node only communicate to a router or hub which requires the minimum transmitting power without interfering the on-body communication between routers.

Because the batteries of on-body nodes can be easily changed, the low power level for operation is not so crucial compared with devices in other types of wireless networks [24]. We do not consider the power consumption as a metric in this paper, and assume the on-body devices have sufficient storage and the resources to run an algorithm with a certain complexity. Thus, all the on-body devices function as router, which means they are capable of relaying packets received from other nodes (end node or another router). Moreover, due to the strict transmitting power requirements as a radio device near human body, its communication range is restricted to a few meters [36].

\subsection{Intra-WBAN Communication}

The intra-body (tier-1) communication includes the data collection and feedback from sensors or actuator to the hub and instructions backwards within a single WBAN. Since our work mainly functions in tier-2, as the basic framework, we assume that the IEEE 802.15.6 standard was used in tier-1. As a low power communication standard specially optimized for WBAN, IEEE 802.15.6 only specifies some regulations in PHY and MAC layers considering the diversity and flexibility of manifold applications.

IEEE 802.15.6 has specified three different physical layers with different frequency bands: Human Body Communication (HBC), Narrow Band (NB) and Ultra-Wide Band (UWB). Since HBC uses electrostatic field communication and UWB technology has high requirements to the hardware, we focus on the NB communication in this paper.

Previous studies show that the path loss near a human body tends to be huge. The path loss exponent ranges from 3 to 4 in LOS transmission and 5 to 7 in NLOS transmission [37], [38], [39]. This leads to high energy consumption for long distance transmission. Besides, due to the high mobility of WBAN nodes caused by postural change and channel variation, the link quality between one node and the hub may be very unstable. Above issues may degrade the performance of the network which is organized in star topology which is specified by IEEE 802.15.6. Meanwhile, the maximum hop counts for intra-body communication is also required to be two in the standard. Thus, the topology can be regarded as a hierarchical one whose maximum node depth is two. Because the implanted devices only communicate with their parents, the link between them is excluded from the hop counts. Since communication in different bands may coexist in a WBAN, we assume each node generates and sends data in its own band which is defined by the application and has the capability to relay packets in all the bands of the group it belongs to as shown in Table.2. For the hub, we regard it is capable of communication in all NB spectrum to collect data from different WBAN applications.

\subsection{Inter-WBAN Communication}

Tier 2 communication includes the data transmission 
between a hub and internet and different hubs. The deployed protocol is usually high rate such as $802.11 \mathrm{a} / \mathrm{b} / \mathrm{g}$. We assume the hub and mobile internet infrastructure have sufficient communication, storage and computational resources which will not be investigated in our work.

The inter-WBAN communication was seldom mentioned in existing works because it does not suffer from the limitations in tier-1 communication and depends on some more mature communication technologies. In this paper, our goal focuses on the tier- 1 communication improvement by exploiting the information exchange and device management among different WBANs, which is not well exploited in previous studies.

\section{SELF-ORgANIZED DYNAMIC ClUSTERING}

\subsection{Spectrum allocation}

The frequency hopping method for WBAN nodes is supported by IEEE 802.15.6. However, the effect may be limited when most of the bandwidth is occupied. Thus, we propose a self-organized dynamic clustering and its multiple access mechanism to mitigate the interference and improve the QoS.

There are $K$ frequency bands supported in the WBANs. An application will use one of them based on the requirements. Let us focus on one band in which there are $N_{k}$ subchannels, where $\mathrm{k}$ is integer from 1 to $K$. We divide them into $M$ equal proportions; each has $N_{c k}$ subchannels, so we have $N_{k}=N_{c k} \times M$.

The multiple WBANs are organized in clusters in our architecture. As the functionality in cellular networks, all the wireless nodes in a cluster share the whole bandwidth of the frequency band which they are working in. This means $N_{k}$ subchannels are to be allocated, a cluster comprises $M$ cells and $N_{c k}$ WBANs (users) in each cell. For the $n$-th cell in a cluster, its allocated channels are the $n$-th channel and every $M$ channels after it, as shown in Fig. 4. Here we define $n$ as the Cell Sequential Number, $C_{\text {seq }}$. When $C_{\text {seq }}$ is given, the activating bands in the cell can be easily determined. On the other hand, in a cell, the channels are sequentially distributed since each of them is distanced $M$ channels away in the frequency domain.

When the cells and clusters are formed based on adjacency, above spectrum allocation method may keep the devices using the same subchannel as far as possible. Because the central device in a cellular cell is the base station, while there are no fixed infrastructures for WBANs communication. The clustering in WBANs is far more challenging. In this context, the self-organized dynamic clustering method is developed, and the FDMA is instinctively supported.

\subsection{Clustering}

In our work, several parameters need to be maintained by each hub:

- $\quad$ Cluster ID. An 8-bit sequential number to identify the cluster in which the hub is working. This value is random generated by the node which initial this cluster.

- Cell ID. An 8-bit sequential number to identify the cell which the hub belongs to. It is determined by its working bands, and its decimal is equal to the

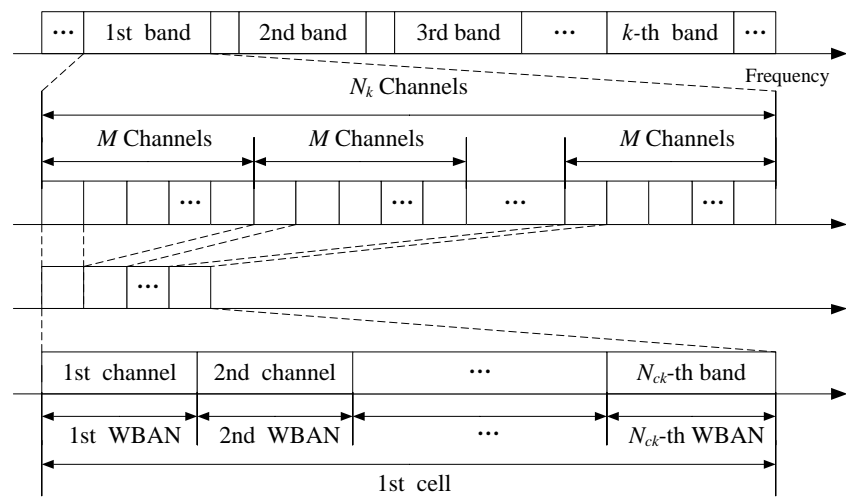

Fig.4. Spectrum allocation in Self-organized Dynamic Clustering

Cseq mentioned above.

- $\quad H u b I D$. An 8-bit sequential number to identify the hub, ranges from 1 to $N_{c k}$. Its decimal value is also the number of nodes in the current cell.

- $\quad N_{\text {node }}$. Number of nodes in the current cell, ranges from 1 to $N_{c k}$.

- $\quad T_{\text {sh. }}$ Time duration for a hub runs the only WBAN in a cell (single hub cell).

- $T_{s h, \max }$. Maximum allowed time duration for a hub runs the only WBAN in a cell.

- $\quad T_{\text {orphan. }}$ Time duration for a hub working in orphan mode, in which it does not belong to any cell or cluster.

- $T_{\text {orpahn, max }}$. Maximum allowed time duration for a hub working in orphan mode

- $R Q_{k}$. An indicator to describe the request QoS level in the $k$-th application band. This value will be used in the TDMA method which will be discussed in next subsection.

Besides, the proposed algorithm is based on the services of each hub as follows:

- All communication bands coverage. The hub is capable of transmitting and receiving packets in any frequency band which its WBAN may use.

- $\quad$ Receiving signal strength indicator (RSSI). For any packet it receives, the receiving power is measured and stored.

- Link quality indicator (LQI). The link quality between the sending and receiving device is estimated for each transmission. The link quality in WBAN covers the path loss, channel history, residual energy and node temperature which relate to different techniques. Since the aim of our work is to mitigate the interference, the RSSI is only considered in the LQI.

- Clear channel assessment (CCA). The hub is capable of identify whether a channel is idle or not for all the channels it supports.

For the self-organized dynamic clustering, a hub has the functions as follows:

- Cluster initial. A hub acts as the first one in a cluster. It generates the Cluster ID and set its Cell ID to 1. This procedure will increase the number of clusters in the WBANs. 
- Cell Initial. A hub acts as the first one in a cell. It inherits the Cluster ID, generates the Cell ID based on the working band and set its Hub ID to 1 .

- Cell Join. A hub joins a cell based on the optimal band, inherit Cluster ID and Cell ID and set Hub ID to the first empty value or $N_{\text {node }}+1$.

- Cell Leave. When a hub finds the average LQI from other cell is better than the value in current cell, it performs leave function and sends a notification. When a hub in the origin cell receives it, it notifies all the hubs in the cell and set the Hub ID of leaving device to empty, $N_{\text {node }}$ decreases by 1 .

- $\quad$ Single cell mode. When a hub acts as the only one in a cell, it works in single hub mode. The $T_{s h}$ will be timing in this mode. When a hub leaves this mode, the $T_{\text {sh }}$ will set 0 .

- Orphan mode. When a hub fails to join adjacent cells, it works in orphan mode. The frequency hopping is used. The $T_{\text {orphan }}$ will be timing in this mode. When a hub leaves this mode, the $T_{\text {orphan }}$ will set 0 .

Since there is no fixed infrastructure in WBAN, the management of a cluster is implemented by all the hubs in the cluster. We call it the cluster management entity (CME) in this paper. In the CME, the information exchange is based on the mobile internet communication, and each hub implements the computation in turn. The CME is responsible of the function of Cell Combine. By periodically communication, if two adjacent clusters find they can be merged by providing enough channels for all the communication in them, the two clusters will be combined to one. In that case, the hubs in the cluster with fewer WBANs will reset their related parameters and implement the Cell Join function. This procedure will decrease the number of clusters in the WBANs. An example of the clustering is shown in Fig.5.

The diagram of Self-organized Dynamic Clustering based on above services and functions is shown in Fig.6. The twice entries for the Cell Join from free Hub ID searching are designed to maximum the efficiency of the spectrum in clusters. The single hub mode is introduced to prevent the case in which an isolated node occupies the channels in a cluster due to its early join, but not being able to share with other WBANs. Orphan mode may prevent the frequent new cluster initialization, which results in high communication overheads. The last issue needs to discuss is the identical Cluster ID in different clusters. It may happen since the parameter is random generate. In this paper, we assume the initial device is aware of the Cluster ID in its neighborhood and generate a unique ID. This procedure can be easily realized by providing GPS information in tier-2 communication or relying on cloud-based architecture.

\subsection{TDMA for SDC}

By the SDC framework mentioned above, the hubs which use the same subchannels are separated as far as possible. However, it may be not far enough. When the density of WBANs is considerable large, a hub may still suffer from the interference from other WBAN device which cannot be ignored. Thus, we introduce a TDMA method for this case.

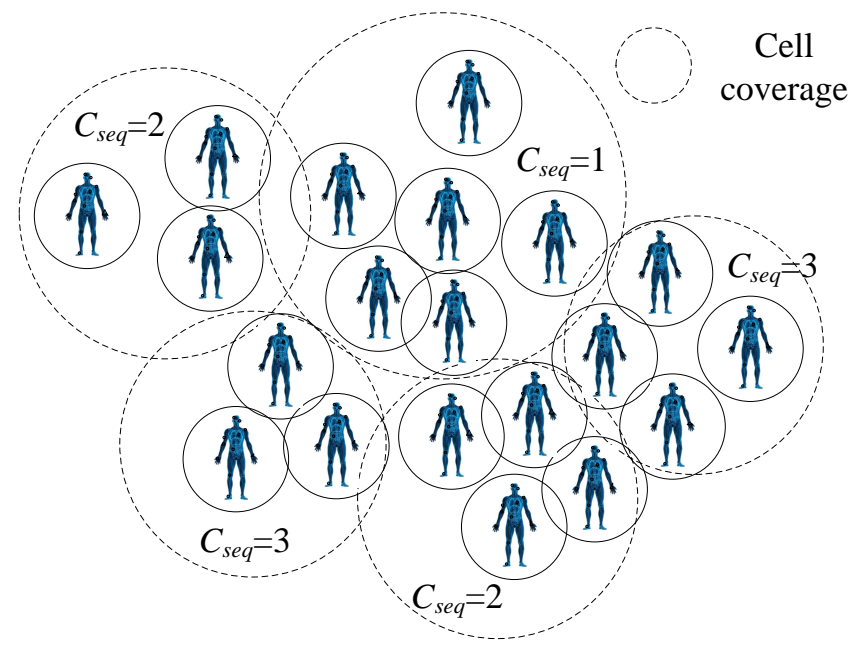

Fig.5. An example of Self-organized Dynamic Clustering, where $M=3$

The superframe structure is enabled in the IEEE 802.15.6 MAC layer, while the allocation mechanism is not specified. To reduce the interference, we allocate the superframe duration based on the parameter of requested QoS for the $k$-th band, $R Q_{k}$ mentioned above. When there are $\mathrm{L}$ applications using this channel, $R_{l}$ and $P_{r l}$ are the data rate and priority of $l$-th application, where $l$ ranges from 1 to $L$, the $R Q_{k}$ is calculated as (1):

\section{$R Q_{k}=\sum_{l=1}^{L} R_{l k} \times P r_{l k} \quad(1)$}

The $R_{l k}$ is determined by the application requirement. Regarding the $P r_{l k}$, since there are four service priorities presented in IEEE 802.15.6, which from low to high are: non-medical services, mixed-medical and non-medical services, general health services, highest priority medical services, we set the $P r_{l k}$ to 1,2,3,4 respectively.

When finding the SIR drop to a pre-defined threshold ( $15 \mathrm{~dB}$ in this paper), a hub will try to allocate the superframe active time with all the interference clusters. Suppose there are $I$ clusters interfere with each other in $K I$ bands, the proportion of active duration for the i-th cluster,

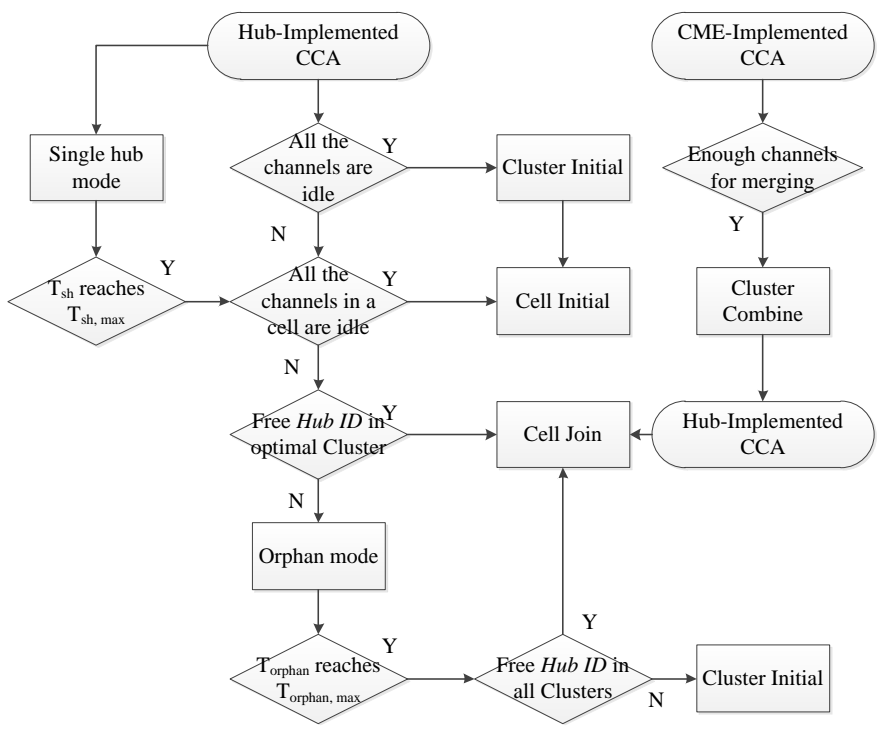

Fig. 6. Diagram for Self-organized Dynamic Clustering 
Di, based on (2)

$$
D_{i}=\frac{\sum_{k=1}^{K I} R Q_{k, i}}{\sum_{i=1}^{I} \sum_{k=1}^{K I} R Q_{k, i}}
$$

\subsection{Inter-WBAN relaying}

Since the multi hop method may provide better performance compared with single hop. A node forwarding protocol based on the inter-WBAN relaying (IWR) is also proposed. Note that the algorithm design is beyond the contents in this paper, we just study the principle for the selection of relay node.

In our scheme, the inter-WBAN communication is allowed between routers from different WBANs but in the same cluster. Because the Cell ID and Hub ID are managed by each device, the channel each router uses can be obtained by calculating the channel sequential number, Channel ID, as (3):

Channel ID $=M \times H u b I D+$ Cell ID (3)

When the IWR is enabled, besides the neighbor routers in its own WBAN, the sending device may extend its next hop candidates to all the neighbor routers in its cluster. We assume each potential relay is aware of the link quality to the sending device and its optimal value to any hub. The summation of them represents the total link cost. The candidate with minimum link cost will be chosen as the next hop node.

Considering the privacy and priority, only the packets from non-medical services and the highest priority medical services are enabled for IMR. The reason is that the former service contains little private information, and the latter one may be life-threatening if the data fails to transmit.

\section{SimUlations}

The performance of SDC was evaluated in this section. The simulation was carried out based on Opnet 14.5. To make a more convincing conclusion, we ran the simulation in two scenarios which were related to different network environments. In scenario 1, the WBANs deploy in high density with medium mobility. The typical scenes include office buildings and mall. And scenario 2 describes the case with extremely high density and low mobility, such as in a stadium or rallies.

As discussed, we assumed the implant device, as end node, was capable of sending packets to an on-body device which provided the best link quality by using in-body communication bands which would not affect the on-body transmission. Above procedure was ignored in our simulation. We only focused on the behaviors of on-body devices which acted as a router and hubs. The hub was on the wrist for each user, while the routers are random deployed as shown in Fig.1. It was impossible to build accurate channel models for each link with such many devices. In this paper, we estimated the related parameters as follows. For the intra-WBAN communication, the distance between a router and hub random ranged from $200 \mathrm{~mm}$ to $800 \mathrm{~mm}$. On the other hand, transmitting distance was the space between two hubs which the communicating nodes belonged to adding a compensation value for height and postural differences, which ranged from 0 to $300 \mathrm{~mm}$. Considering the path loss model, we assumed it might be LOS or NLOS
TABLE 3

SIMULATION PARAMETERS

\begin{tabular}{lcc}
\hline \hline \multicolumn{1}{c}{ Parameters } & Scenario 1 & Scenario 2 \\
\hline WBAN numbers & 120 & 240 \\
Simulation area & $36 \mathrm{~m} * 36 \mathrm{~m}$ & $36 \mathrm{~m} * 36 \mathrm{~m}$ \\
Duration & $30 \mathrm{mins}$ & $30 \mathrm{mins}$ \\
Mobility & random from $3 \sim 7 \mathrm{~m} / \mathrm{s}$ & random from $0.1 \sim 0.5 \mathrm{~m} / \mathrm{s}$ \\
Number of bands a WBAN uses & 6 & 6 \\
Applications in a band & random from $1 \sim 3$ & random from $1 \sim 3$ \\
Routers per application & random from $1 \sim 3$ & random from $1 \sim 3$ \\
Channels in a band $\left(N_{k}\right)$ & 20 & 20 \\
Number of cells per cluster $(M)$ & 4 & 4 \\
Channels in each cluster $\left(N_{c k}\right)$ & 5 & 5 \\
Packets with different priorities & $1: 1: 1: 1$ & $1: 1: 1: 1$ \\
Retransmitting times & 3 & 3 \\
$T_{\text {sh,max }}$ & $60 \mathrm{~s}$ & $60 \mathrm{~s}$ \\
$T_{\text {orphan,max }}$ & $1 \mathrm{~s}$ & $1 \mathrm{~s}$ \\
\hline \hline
\end{tabular}

in even chance. The path loss exponent was random from 3 to 4 for LOS and 5 to 7 for NLOS. For a certain router, each service it provided worked in a random NB band for on-body communication listed in Table.2. In our simulation, since the diversity of data rate in different application, we measure the data rate by the percentage of maximum data rate supported in the band it was using. The data rates ranged from $10 \%$ to $90 \%$ of the maximum rate, with interval of $20 \%$. The transmit power also varied according to the application requirements. In our work, we assumed the sending device had the knowledge of the path loss and the required receiving signal strength, and then transmitted the packet in minimum power possible. Besides mentioned above, other general simulation parameters are shown in Table. 3.

Because SDC was designed for interference mitigation, SIR was measured for performance evaluation. Besides, the QoS was also considered in terms of the packet delivery ratio and packet delay. The performance was compared with the non cluster architecture which used IEEE 802.15.6 for tier 1 communication and the frequency hopping was enabled. Meanwhile, we also deployed the proposed IWR in SDC and tested its performance.

The SIRs for different methods in both scenarios were shown Fig.7. It could be concluded that the self-organized dynamic clustering and its multiple access mechanism were effective in the SIR mitigation. SDC had the best performances, with an average $5.3 \mathrm{~dB}$ and $3.9 \mathrm{~dB}$ higher than IEEE 802.15.6 in scenario 1 and 2, separately. It benefited from the longer distance between the WBAN nodes which worked in the same subchannel by clustering. We could also see that when the inter-WBAN relaying was activated, the effect on interference mitigation was counteracted a bit. The reason of this might be that the relaying node from other WBAN may locate nearer to a signal or interference source which was deployed in another cluster. The average improvement on SIR decreased to $4.0 \mathrm{~dB}$ and $2.3 \mathrm{~dB}$ on average. Besides, the faster decline of all simulated methods indicated the effects of node density. On the other hand, our mechanism could make more sense in crowded situation since the SIR without clustering had fallen approximately to $3 \mathrm{~dB}$ which would degenerate the network performance seriously. 
As the indicator of reliability, the packet delivery ratio was illustrated in Fig. 8. SDC improved this value for $9.3 \%$ and $7.0 \%$ on average compared with IEEE 802.15.6. On one hand, the higher SIR resulted in better Signal-to-Interference-plus-Noise-Ratio (SINR) which reduced the error rate. On the other, the determined band (based on Eq. (3)) might reduce the noise impact on frequency distortion. Moreover, it was shown that the IWR could further improve reliability, which was an instinctive advantage of multi hop communication. On average, SDC improved PDR by $9.2 \%$ and $9.7 \%$ in scenario 1 and 2 . The value for SDC with IWR was $16.5 \%$ and $11.7 \%$. Focusing on the slopes of PDR variation in both sub-figures, one could see that the WBAN mobility had a bigger impact on the PDR and our methods were effective in both scenarios.

In Fig.9, because packet delay had a stronger relationship to the response time of a service or command, it was chosen rather than end-to-end delay to be studied. What happened in both scenarios seemed similar. SDC was able to reduce the latency effectively no matter whether IWR was enabled. This was partially because of the improved bit error rate which was analyzed before, at the same time, the organized

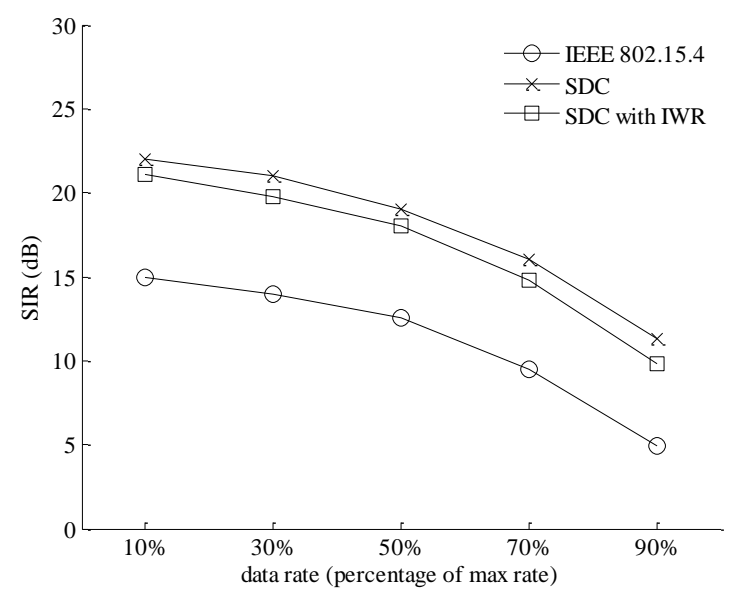

(a) SIRs in scenario 1

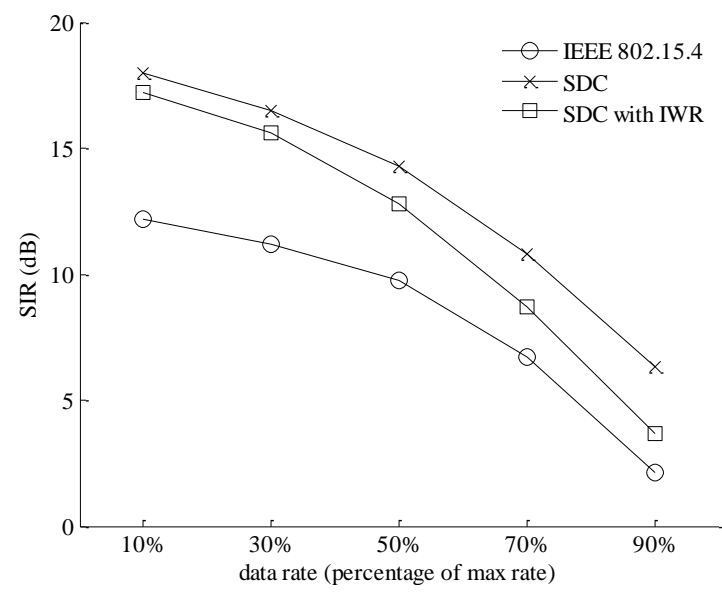

(b) SIRs in scenario 2 frequency and superframe allocation could reduce the chance of collision. The figures also indicated that when the data rate was low, IWR had better performance while the single SDC had less latency in high data rate case. The reason might be that the improvement was balanced by the rising channel occupancy in multi hop scheme.

\section{CONCLUSIONS}

The WBAN provides continuous data sensing and gathering around human body without discomforting the user for various medical and non-medical applications. As it is getting more and more popular with the development of some fundamental technologies, WBAN may be ubiquitous in our daily life in the future. A well designed management architecture is needed to reduce the influences caused by the coexistence of multiple, even massive WBANs. In this paper, we propose a Self-organized Dynamic Clustering method and its multiple access mechanism to mitigate the interference and improve the communication QoS for multiple WBANs environment. To the best of our knowledge, this is the first paper which focuses

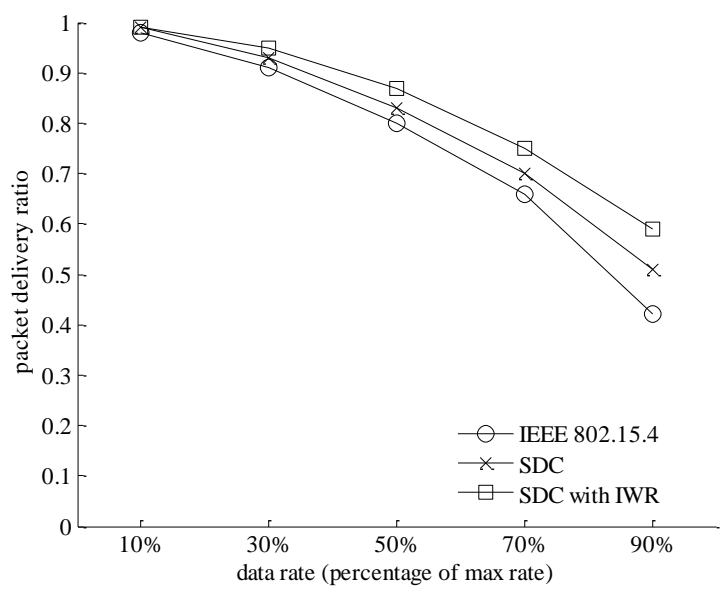

(a) Packet delivery ratios in scenario 1

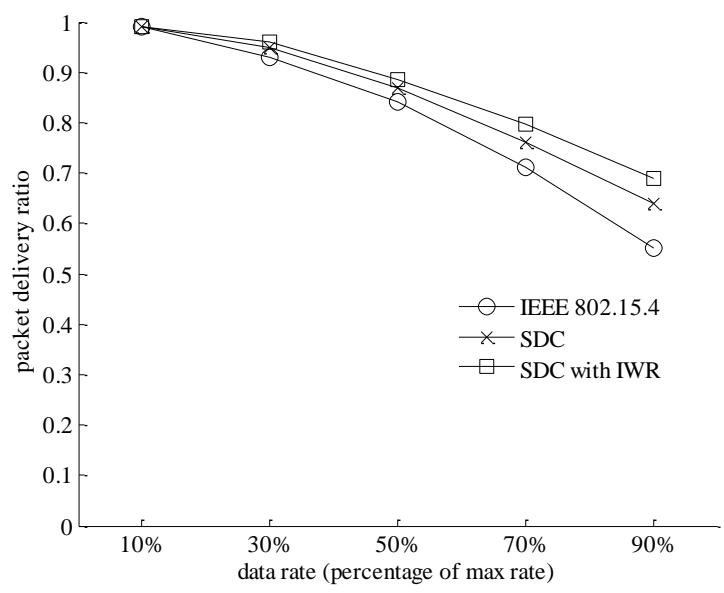

(b) Packet delivery ratios in scenario 2 
on the spectrum allocation for multiple WBANs. In SDC, the clustering is self-organized by the hubs' operation and communication in tier 2. FDMA is instinctively supported by cluster framework, while the TDMA is based on the superframe structure. Furthermore, we propose an interWBAN relaying protocol by allowing the inter-WBAN communication in a cluster. The simulation results show that the SDC and SDC with IWR achieve better SIR, higher PDF and lower packet latency.

Since our study on the multiple WBANs is still at the beginning phase, our future work may include the improvement on existing algorithm, protocol design on node level, cloud-based method investigation and so on.

\section{ACKNOWLEDGMENT}

This paper is supported by the National Natural Science Foundation of China (61401310, 61501326, 61731006, and 61271411). It also supported by China Scholarship Council (201608120043), Tianjin Research Program of Application Foundation and Advanced Technology(15JCZDJC31500), and Tianjin Science Foundation(16JCYBJC16500).

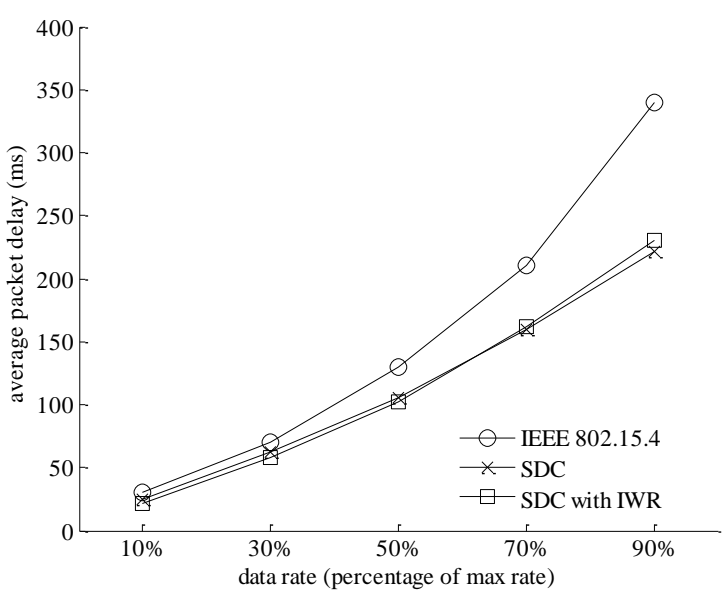

(a) SIRs in scenario 1

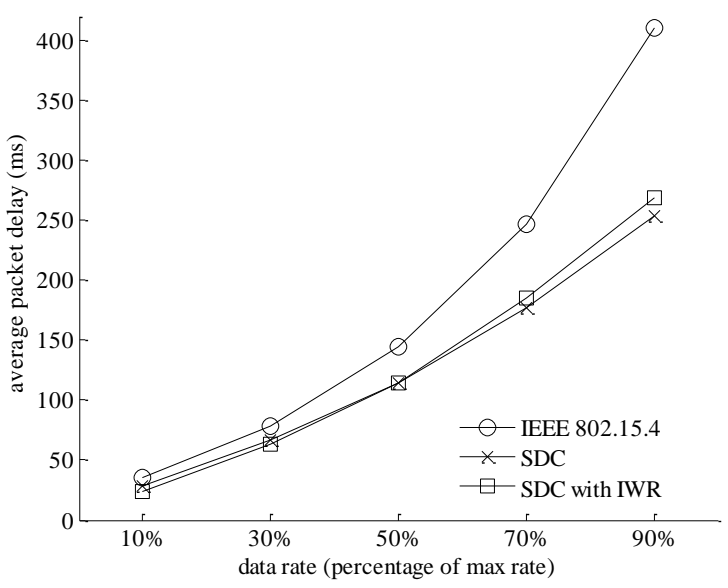

(b) SIRs in scenario 2

\section{REFERENCES}

[1] Nigussie, Ethiopia, Teng Xu, and Miodrag Potkonjak. "Securing wireless body sensor networks using bijective function-based hardware primitive." Intelligent Sensors, Sensor Networks and Information Processing (ISSNIP), 2015 IEEE Tenth International Conference on. IEEE, 2015.

[2] Cavallari, Riccardo, et al. "A survey on wireless body area networks: Technologies and design challenges." IEEE Communications Surveys \& Tutorials 16.3 (2014): 1635-1657.

[3] Gravina, Raffaele, et al. "Multi-sensor fusion in body sensor networks: State-of-the-art and research challenges." Information Fusion 35 (2017): 68-80.

[4] Alrige, Mayda, and Samir Chatterjee. "Toward a taxonomy of wearable technologies in healthcare." International Conference on Design Science Research in Information Systems. Springer, Cham, 2015.

[5] Wei, Y. U., Q. I. N. G. S. O. N. G. Fei, and L. I. J. U. A. N. He. "Sports motion analysis based on mobile sensing technology." International Conference on Global Economy, Finance and Humanities Research (GEFHR 2014). 2014.

[6] Solanas, Agusti, et al. "Smart health: a context-aware health paradigm within smart cities." IEEE Communications Magazine 52.8 (2014): 74-81.

[7] Hiremath, Shivayogi, Geng Yang, and Kunal Mankodiya. "Wearable Internet of Things: Concept, architectural components and promises for person-centered healthcare." Wireless Mobile Communication and Healthcare (Mobihealth), 2014 EAI 4th International Conference on. IEEE, 2014.

[8] Swan, Melanie. "Sensor mania! the internet of things, wearable computing, objective metrics, and the quantified self 2.0." Journal of Sensor and Actuator Networks 1.3 (2012): 217-253.

[9] Chen, Min, et al. "Body area networks: A survey." Mobile networks and applications 16.2 (2011): 171-193.

[10] Movassaghigilani, S., Mehran Abolhasan, and Justin Lipman. "A review of routing protocols in wireless body area networks." Journal of Networks (2013).

[11] Awan, Khalid, Kashif Naseer Qureshi, and Mehwish. "Wireless body area networks routing protocols: a review." Indonesian Journal of Electrical Engineering and Computer Science 4.3 (2016): 594-604.

[12] Wang, Yue. "Linear least squares localization in sensor networks." Eurasip journal on wireless communications and networking 2015.1 (2015): 51.

[13] Liang, Qilian, and Qingchun Ren. "Energy and mobility aware geographical multipath routing for wireless sensor networks." Wireless Communications and Networking Conference, 2005 IEEE. Vol. 3. IEEE, 2005.

[14] Movassaghi, Samaneh, et al. "Wireless body area networks: A survey." IEEE Communications Surveys \& Tutorials 16.3 (2014): 1658-1686.

[15] Bluetooth Core Specification, Core Version 5.0 (2016). https://www.bluetooth.com/specifications/bluetooth-core-specification

[16] IEEE Std 802.15.4-2015 (Revision of IEEEStd 802.15.4-2011) - IEEE Standard for Low-Rate Wireless Networks. https://standards.ieee.org/findstds/standard/802.15.4-2015.html

[17] Lai, Xiaochen, et al. "A survey of body sensor networks." Sensors 13.5 (2013): 5406-5447.

[18] IEEE Standard for Local and metropolitan area networks - Part 15.6: Wireless Body Area Networks, 2012

[19] Kwak, Kyung Sup, Sana Ullah, and Niamat Ullah. "An overview of IEEE 802.15.6 standard." Applied Sciences in Biomedical and Communication Technologies (ISABEL), 2010 3rd International Symposium on. IEEE, 2010. 
[20] Ullah, Sana, Manar Mohaisen, and Mohammed A. Alnuem. "A review of IEEE 802.15. 6 MAC, PHY, and security specifications." International Journal of Distributed Sensor Networks 9.4 (2013): 950704.

[21] Cordeiro, Carlos. "Use cases, applications and requirements for BANs." Doc.: IEEE802. 15-07-0564-00-0ban (2007).

[22] Yang, Wen-Bin, and Kamran Sayrafian-Pour. "Interference mitigation for body area networks." Personal Indoor and Mobile Radio Communications (PIMRC), 2011 IEEE 22nd International Symposium on. IEEE, 2011.

[23] Liang, Xuedong, llangko Balasingham, and Sang-Seon Byun. "A reinforcement learning based routing protocol with QoS support for biomedical sensor networks." Applied Sciences on Biomedical and Communication Technologies, 2008. ISABEL'08. First International Symposium on. IEEE, 2008.

[24] Filipe, Luis, et al. "Wireless body area networks for healthcare applications: Protocol stack review." International Journal of Distributed Sensor Networks 11.10 (2015): 213705.

[25] Liang, Xuedong, and llangko Balasingham. "A QoS-aware routing service framework for biomedical sensor networks." Wireless Communication Systems, 2007. ISWCS 2007. 4th International Symposium on. IEEE, 2007.

[26] Djenouri, Djamel, and Ilangko Balasingham. "LOCALMOR: Localized multi-objective routing for wireless sensor networks." Personal, Indoor and Mobile Radio Communications, 2009 IEEE 20th International Symposium on. IEEE, 2009.

[27] Quwaider, Muhannad, and Yaser Jararweh. "Cloudlet-based efficient data collection in wireless body area networks." Simulation Modelling Practice and Theory 50 (2015): 57-71.

[28] Razzaque, Md Abdur, Choong Seon Hong, and Sungwon Lee. "Datacentric multiobjective QoS-aware routing protocol for body sensor networks." Sensors 11.1 (2011): 917-937.

[29] Kim, Seungku, and Byung Kwon Song. "A prioritized resource allocation algorithm for multiple wireless body area networks." Wireless Networks 23.3 (2017): 727-735.

[30] Dong, Jie, and David Smith. "Cooperative body-area-communications: Enhancing coexistence without coordination between networks." Personal Indoor and Mobile Radio Communications (PIMRC), 2012 IEEE 23rd International Symposium on. IEEE, 2012.

[31] de Silva, Buddhika, Anirudh Natarajan, and Mehul Motani. "Inter-user interference in body sensor networks: Preliminary investigation and an infrastructure-based solution." 2009 Body Sensor Networks. IEEE, 2009.

[32] Lazzi, Gianluca. "Thermal effects of bioimplants." IEEE Engineering in Medicine and Biology Magazine 24.5 (2005): 75-81.

[33] Tang, Qinghui, et al. "Communication scheduling to minimize thermal effects of implanted biosensor networks in homogeneous tissue." IEEE Transactions on Biomedical Engineering 52.7 (2005): 1285-1294.

[34] Tang, Qinghui, et al. "TARA: thermal-aware routing algorithm for implanted sensor networks." International Conference on Distributed Computing in Sensor Systems. Springer, Berlin, Heidelberg, 2005.

[35] Yuce, Mehmet R., et al. "A MICS band wireless body sensor network." Wireless Communications and Networking Conference, 2007. WCNC 2007. IEEE. IEEE, 2007.

[36] C95.1-2005-IEEEStandard for Safety Levels with Respect to Human Exposure to Radio Frequency Electromagnetic Fields, $3 \mathrm{kHz}$ to $300 \mathrm{GHz}$, 1999.

[37] Latré, Benoît, et al. "Networking and propagation issues in body area networks." Proceedings of the 11th Symposium on Communications and Vehicular Technology in the Benelux, SCVT 2004. 2004.

[38] Braem, Bart, et al. "The need for cooperation and relaying in short-range high path loss sensor networks." Sensor Technologies and Applications,
2007. SensorComm 2007. International Conference on. IEEE, 2007.

[39] Jia, Min, et al. "Downlink Design for Spectrum Efficient IoT Network." IEEE Internet of Things Journal PP.99(2017):1-8.

Jiasong $\mathrm{Mu}$ received the BS degree in electric information engineering from Harbin Institute of Technology, China, in 2005, the MS degree in signal and information processing from Tianjin University, China, in 2007, and the $\mathrm{PhD}$ degree in electronic circuit and system from Tianjin University, China, in 2012. He has been an associate professor at the College of Electronic and Communication Engineering, Tianjin Normal University, China, since September, 2014. His research interests include wireless sensor network, body area networks, 5G, smart grid and wireless communication. He is the TPC co-chair of the International Conference on Communications, Signal Processing, and Systems from 2015 to 2018.

Robert Stewart. is currently the Professor of Signal Processing at the University of Strathclyde, Scotland, UK in the Dept of Electronic and Electrical Engineering, was previously Department Chair and Head of Department from 2014-2017. In recent years his research work has been on digital communications and software defined radio, with specific interest in model-based design, spectrum sharing and TV white space and on radio standards. Bob has also worked extensively on rural communications issues in both UK and in Africa and is currently PI on an EPSRC funded project (EP/P029698/1) on 'Enabling Affordable 'Internet Access using Dynamic Spectrum Management'. Since 1997 Bob has been engaged as a visiting Professor at the University of California, Los Angeles (UCLA) Extension School. Over his career Bob has authored many academic and technical papers, as well as 3 textbooks. In 2004 Bob was a co-founder of the startup digital communications company Steepest Ascent Ltd which was later acquired by MathWorks in 2013. Bob has a PhD from the University of Strathclyde.

Liang Han received the M.S. and Ph.D. degree in communication and information systems, both from University of Electronic Science and Technology of China, Chengdu, China, in 2010 and 2013, respectively. Since 2014, he has been with the College of Electronic and Communication Engineering, Tianjin Normal University. From 2016 to 2017, he was a visiting scholar in the Department of Electrical Engineering at the University of Texas at Arlington. His current research interests include D2D communications, full-duplex communications and V2X communications.

David Crawford After spending almost 20 years in industry in a number of technical roles related to telecommunications and digital signal processing, Dr David Crawford has been running the Centre for White Space Communications at the University of Strathclyde since its inception in March 2011. During this time, he has led the Centre to its current position of a strong international presence in dynamic spectrum access and white space technologies, and worked with UK Govt offices such as Ofcom, tier one industry partners (Microsoft, Motorola, BBC, British Telecom, Cisco), alongside managing international collaboration engagement with partners in Africa (Kenya, Malawi, Ghana and Zambia on affordable internet access projects. Previously Dr Crawford has held senior industry positions to Epson, Motorola, and Ensigma, David has a PhD from the University of Strathclyde awarded in 29916, and also has a BSc, MSc and MBA. 